Article

\title{
Optimal Harvest Time for Preventing Hot Pepper Seed Browning during Cold Storage Is Associated with Seed Maturity
}

\author{
Me-Hea Park ${ }^{1, *}$, Jung-Soo Lee ${ }^{1}$, Eun-Young Yang ${ }^{2}$, Gyung-Ran Do ${ }^{3}$ and Yoon-Pyo Hong ${ }^{1, *}$ \\ 1 Postharvest Research Division, National Institute of Horticultural \& Herbal Science, Wanju 55365, Korea; \\ ljs808@korea.kr \\ 2 Vegetable Research Division, National Institute of Horticultural \& Herbal Science, Wanju 55365, Korea; \\ yangyang2@korea.kr \\ 3 Planning and Coordination Division, National Institute of Horticultural \& Herbal Science, \\ Wanju 55365, Korea; microdo@korea.kr \\ * Correspondence: poemmich@korea.kr (M.-H.P.); hongyp0113@korea.kr (Y.-P.H.); \\ Tel.: +82-63-238-6512 (M.-H.P.)
}

Received: 21 October 2020; Accepted: 25 November 2020; Published: 26 November 2020

\begin{abstract}
Chilling injury (CI), which causes seed browning in pepper, may arise following long-term cold storage, and is a major cause of postharvest losses. To explore potential strategies of minimizing the associated postharvest losses, the present study investigated the optimal pepper harvest time that could reduce levels of seed browning, in addition to the relationship between fruit maturity and seed browning. Fruits harvested 15 days after flowering (DAF) were sensitive to cold storage at $4{ }^{\circ} \mathrm{C}$ and exhibited $100 \%$ seed browning (CI index, 4.0); in contrast, the seed browning rate of fruits harvested 35 DAF was $10 \%$ (CI index, 0.4$)$ within 7 days of cold storage. Seed antioxidant activity was higher in seeds harvested at early stages (15 DAF to $20 \mathrm{DAF}$ ) than in seeds harvested at later stages (40 DAF to 50 DAF) at the beginning of storage. Pericarps of fruit harvested at 50 DAF exhibited the highest antioxidant activity. Lipoxygenase, catalase, and peroxidase activity, and the expression levels of cell wall-related genes, pectin methylesterase-like protein, and endo- $\beta-1,4$-glucanase were higher in seeds of immature fruit harvested 15 DAF than in seeds of mature fruit harvested 35 DAF. The seeds of the fruit harvested 35 DAF were fully developed with the seed coat separated from the endosperm and did not turn brown under low-temperature storage. The lack of seed browning observed in mature fruit under low-temperature storage could be attributed to physical protection provided by the seed coat rather than cold stress resistance conferred by antioxidants.
\end{abstract}

Keywords: chilling injury; pepper; seed browning; maturity; harvest time

\section{Introduction}

Hot pepper (Capsicum annuum L.) is a subtropical crop that is susceptible to chilling injury (CI). $\mathrm{CI}$ is observed in hot pepper subjected to $<7-10{ }^{\circ} \mathrm{C}$ for $1-2$ weeks $[1,2]$. The symptoms of $\mathrm{CI}$ in pepper include seed browning, surface pitting, shrinkage, and calyx discoloration [2-4]. However, chilling injury severity could vary across cultivars, fruit maturity levels, and exposure times [5]. Postharvest $\mathrm{CI}$ decreases pepper fruit quality, which causes yield losses during cold storage and in the course of market distribution [6-8]. Nevertheless, cold storage is an effective postharvest management technology for the maintenance of horticultural crop quality and the suppression of respiration [2]; consequently, it is critical to investigate appropriate strategies of storing and maintaining the quality of such sensitive and commercially important crops. 
Seed browning is major symptom of CI in hot pepper [9]. Severe seed browning has been observed in hot pepper stored at $5{ }^{\circ} \mathrm{C}$, which has been attributed to invisible cell damage and the release of free phenolic compounds $[9,10]$. Under severe CI, the exocarp turns brown or black, and the epidermis and hypodermal cortical cells undergo necrosis [11]. Browning symptoms could be minimized by reducing storage and distribution periods. In previous studies exploring mechanisms of reducing seed browning, seed browning was significantly inhibited by methyl jasmonate (MeJA) but induced by methyl salicylate during storage at $2{ }^{\circ} \mathrm{C}$; in addition, treatment with MeJA inhibited seed browning by increasing endogenous jasmonic acid (JA) production and upregulating the expression of antioxidant-related genes in pepper fruit $[2,10]$.

It has been proposed that $\mathrm{CI}$ induces oxidative stress responses and that antioxidants protect the fruit against cold-induced damage [12]. Similarly, CI symptoms have been attributed to cell membrane damage by reactive oxygen species (ROS) generated from lipid peroxidation [5,6]. CI increases membrane permeability and the leakage of ions from the cytoplasm into the intercellular spaces [13], and results in ultrastructural changes to the membrane [14].

However, CI sensitivity also depends on crop maturity. Mature persimmon and tomato fruit were relatively less sensitive to CI than immature fruit [15]. In addition, green bell pepper was more sensitive to surface pitting than mature pepper fruit [16]. Chilling stress responses in the form of seed browning have also been observed in young eggplant fruit, with young seeds being more susceptible to chilling-induced browning than old seeds $[5,17]$. The determination of optimal harvest time of subtropical crops such as pepper could be an effective method of reducing postharvest losses associated with CI; however, few studies have examined the correlation between harvest time, based on fruit maturity, and seed browning under cold storage. Most previous studies have only explored the physiological changes that occur in pepper fruit exposed to CI [18], the metabolite changes in brown seeds under various storage temperatures [9], and seed browning based on different early harvest times following low-temperature storage [5].

Here, to explore a strategy for reducing postharvest losses and maintain hot pepper quality during cold storage and in the course of market distribution, we investigated the optimal harvest time for preventing seed browning induced by CI. We examined changes in fruit quality, antioxidant activity, gene expression, and anatomy at different stages of fruit maturity, namely, 15, 20, 25, 30, 35, 40, 45, and 50 days after flowering (DAF), following cold storage. The results of the present study could not only enhance our understanding of the mechanism of seed browning following CI but also facilitate the formulation of effective postharvest management approaches.

\section{Materials and Methods}

\subsection{Plant Materials and Storage Conditions}

Pepper (Capsicum annuum L. "Nockgwang") plants were grown in the greenhouses of the National Institute of Horticultural and Herbal Science, Wanju, Republic of Korea. Pepper "Nockgwang" seeds (Dongbu Farm Co., Ltd. Seoul. Korea) was sown into a plug tray with filling soil (Baroker, Seoul Bio, Seoul, Korea) for seedlings. After sowing, it was grown for 10 weeks after germination in dark conditions at a temperature of $30{ }^{\circ} \mathrm{C}$ and relative humidity of $85 \%$ and then planted in the field.

The fruits were harvested at 15, 20, 25, 30, 35, 40, 45, and 50 DAF (Figure 1A). Fruits were harvested from different plants at each time point. Harvested fruit were washed with water containing $200 \mu \mathrm{L} \mathrm{L}^{-1}$ sodium hypochlorite and air-dried at room temperature $\left(25 \sim 28^{\circ} \mathrm{C}\right)$. They were then either packed in a plastic tray with plastic film and stored at $4{ }^{\circ} \mathrm{C}$ for 15 days, and followed by 4 days at $20^{\circ} \mathrm{C}$. The relative humidity was maintained at $85 \pm 5 \%$. Fruits were evaluated following the storage. Twenty samples per treatment were used for quality evaluation at each time point. Seeds and pericarp from 20 samples per treatment at each time point were excised for antioxidant activity analyses and RNA extraction, flash-frozen in liquid nitrogen, and stored at $-80^{\circ} \mathrm{C}$. 

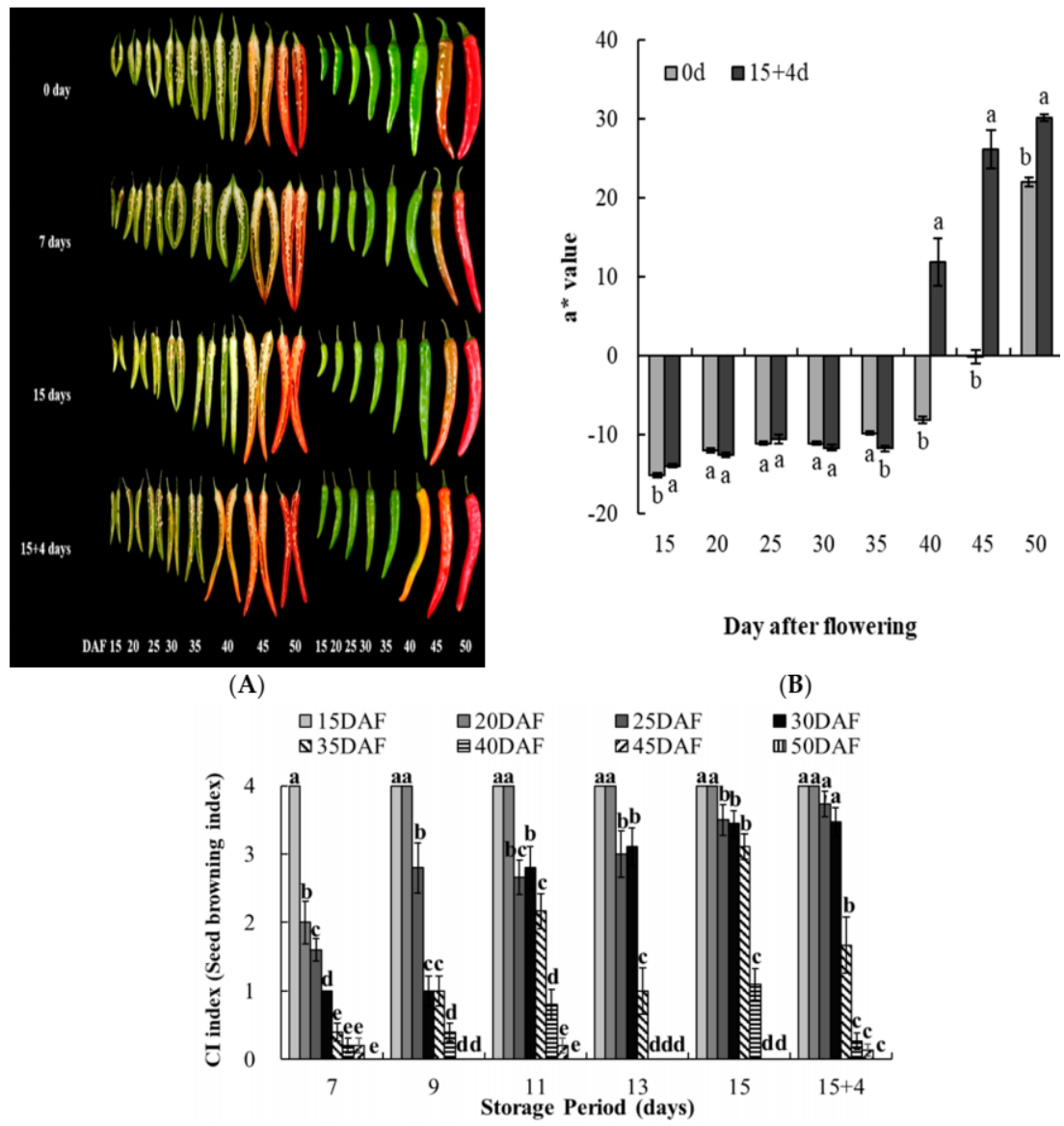

(C)

Figure 1. Changes in pepper fruit skin color and seed browning during storage. (A) Photograph of seeds and pericarp of pepper fruit. (B) Changes in skin color a value (redness) in fruit harvested at different times 0 day and 15 days after cold storage and maintained at $20{ }^{\circ} \mathrm{C}$ for 4 days $(15+4 \mathrm{~d})$. (C) Chilling injury (CI, Seed browning) index. Data are means \pm standard error (SE) $(n=20)$. Different letters indicate significant difference among treatments at the same storage time according to Duncan's multiple range test (DMRT). $p<0.05$. Fruits were harvested at 15, 20, 25, 30, 35, 40, 45, and 50 days after flowering (DAF), stored for 15 days at $4{ }^{\circ} \mathrm{C}$ and for 4 days at $20{ }^{\circ} \mathrm{C}(15+4)$.

\subsection{Fruit Quality Evaluation}

For the firmness assay, 20 pepper fruits were selected from the different harvest time treatments and two portions of each fruit were measured with a texture analyzer (TA Plus Lloyd Instruments Ltd., Fareham, Hampshire, UK) fitted with a plunger head $3 \mathrm{~mm}$ in diameter. Its operating speed was $0.5 \mathrm{~mm} \mathrm{~s}^{-1}$. Fifteen fruit samples were used to measure total soluble solids (TSS) content and titratable acidity (TA) with a digital refractometer (PAL-1; Atago Co. Ltd., Saitama, Japan), and an auto $\mathrm{pH}$ titrator (TitroLine Easy; Schott Instruments $\mathrm{GmbH}$, Mainz, Germany). TA was expressed in $\mathrm{g}$ acetic acid/100 g sample juice. For the fruit skin color assessment, 15 samples per treatment were measured and two places in the middle portion of each fruit were examined using a color difference meter (Model CR-400, Minolta, Osaka, Japan) based on Hunter's a* scale.

The $\mathrm{CI}$ index was calculated according to the degree of seed browning, which was visually rated as follows: $0=$ no symptom; $1=<25 \% ; 2=25-50 \% ; 3=50-75 \% ; 4+=>75 \%$. The CI index was calculated based on the following formula [19]:

$\mathrm{CI}$ index (Seed browning index $)=[\Sigma$ (chilling injury grade $) \times($ number of fruit at the chilling injury grade) $] /$ (total number of fruits in the treatment) 


\subsection{Antioxidant, Total Phenol Analysis}

Each sample was freeze-dried and pulverized. The seed powder $(100 \mathrm{mg})$ or pericarp powder $(50 \mathrm{mg})$ was extracted twice in $1 \mathrm{~mL}$ of $70 \%(v / v)$ ethanol with shaking for $1 \mathrm{~h}$ at $20 \pm 2{ }^{\circ} \mathrm{C}$. The extracts were centrifuged at $4{ }^{\circ} \mathrm{C}$ at $12,000 \times g$ for $15 \mathrm{~min}$. The supernatant was passed through a $0.2-\mu \mathrm{m}$ Teflon ${ }^{\circledR}$ (PTFE) filter and the filtered extracts were used in the antioxidant activity assay.

The total polyphenol content was measured according to the method of Singleton and Rossi [20], with modifications. Briefly, $100 \mu \mathrm{L}$ extract or standard was mixed with $100 \mu \mathrm{L}$ of $1 \mathrm{~N}$ Folin-Ciocalteu reagent for $3 \mathrm{~min}$ and then $1 \mathrm{~mL}$ of $2 \%(w / v)$ sodium carbonate solution was added. The mixture was incubated for $30 \mathrm{~min}$ and its absorbance was read at $726 \mathrm{~nm}$ using a microplate reader (EPOCH2, BioTek Instruments Inc., Winooski, VT, USA). The output was expressed as mM gallic acid equivalents (GAE)/g DW.

1,1-Diphenyl-2-picrylhydrazyl (DPPH) radical scavenging activity was assessed according to the method of Dietz et al. [21]. Twenty microliters of extract was mixed with $180 \mu \mathrm{L}$ of $0.18 \mathrm{mM} \mathrm{DPPH}$ and briefly vortexed. The samples were set aside for $20 \mathrm{~min}$ and the absorbance was measured at $515 \mathrm{~nm}$ using a microplate reader. DPPH-free methanol served as the blank reference. The absorbance was converted to DPPH radical scavenging activity. 2,2-azino-bis-3-ethylbenzothiazoline-6-sulfonic acid (ABTS) radical scavenging activity was evaluated according to the method of Re et al. [22], with modifications. In brief, $7 \mathrm{mM}$ ABTS stock solution was prepared by combining $7 \mathrm{mM}$ ABTS with $2.45 \mathrm{mM}$ potassium persulfate and the mixture was stored in the dark for $16 \mathrm{~h}$. The solution was adjusted with ethanol until the absorbance at $734 \mathrm{~nm}$ was $0.7 \pm 0.02$ at ambient temperature $\left(25 \pm 2{ }^{\circ} \mathrm{C}\right)$. Subsequently, $20 \mu \mathrm{L}$ of each extract was mixed with $180 \mu \mathrm{L}$ of the ABTS+ solution and the absorbance at $734 \mathrm{~nm}$ was measured using a microplate reader at ambient temperature $\left(25 \pm 2{ }^{\circ} \mathrm{C}\right)$ after $10 \mathrm{~min}$. The ABTS radical scavenging activity was expressed as Trolox equivalent antioxidant capacity $(\mu \mathrm{mol} \mathrm{TE} / \mathrm{g} \mathrm{DW})$.

\subsection{Gene Expression Analysis}

All samples were frozen in liquid nitrogen and pulverized to a fine powder in a mortar and pestle. Total RNA was extracted from the pericarp tissue using the RNeasy plant mini kit (Qiagen, Valencia, CA, USA). Five hundred nanograms of total RNA was used to synthesize cDNA in a QuantiTect reverse transcription kit (Qiagen, Valencia, CA, USA). The product was amplified using $\mathrm{QQ}^{\mathrm{TM}} \mathrm{SYBR}$ Green Supermix and specific primers (Supplementary Table S1). Quantitative real-time polymerase chain reaction (RT-qPCR) was performed in a CFX96 Touch $^{\mathrm{TM}}$ real-time PCR detection system (Bio-Rad Laboratories, Hercules, CA, USA). The PCR was programmed as follows: $95{ }^{\circ} \mathrm{C}$ for $30 \mathrm{~s}$, followed by 40 cycles of $95^{\circ} \mathrm{C}$ for $10 \mathrm{~s}$, and $55^{\circ} \mathrm{C}$ or $58^{\circ} \mathrm{C}$ for $40 \mathrm{~s}$. Relative gene expression was calculated using the $2^{-\Delta \Delta C t}$ method of Livak and Schmittgen [23] and normalized against the expression levels of the housekeeping genes, Actin and eukaryotic initiation factor 4E (eIF4E). The qRT-PCR analysis was conducted using at least 3 and 2 biological and technical replicates, respectively.

\subsection{Anatomical Analysis under Light Microscopy and Scanning Electron Microscopy}

Stereomicroscopy (SM): Live seeds collected at each harvest time were observed under a helium stereomicroscope (Stemi-2000; Carl Zeiss AG, Oberkochen, Germany).

Light microscopy (LM): The procedures used were modified from Clement et al. [24]. Seeds were fixed for $24 \mathrm{~h}$ in $2.5 \%(v / v)$ glutaraldehyde in $0.1 \mathrm{M}$ phosphate buffer ( $\mathrm{pH} 7.2)$ with $4 \%(w / v)$ sucrose. After three 30-min rinses in phosphate buffer, the specimens were post-fixed for $4 \mathrm{~h}$ with $1 \%(w / v)$ $\mathrm{OsO}_{4}$ in the buffer plus $4 \%(w / v)$ sucrose. The samples were then rinsed thrice with buffer for $30 \mathrm{~min}$ each time, dehydrated in an alcohol gradient series, immersed in propylene oxide, and embedded in Epon epoxy resin. Semi-thin sections $(2.5 \mu \mathrm{m})$ were prepared using a ultramicrotome, mounted on glass microscope slides, and subjected to periodic acid-Schiff (PAS) polysaccharide-specific treatment. PAS-positive reactions were red in color. The thin sections were immersed in $1 \%(w / v)$ periodic acid for 
$30 \mathrm{~min}$, and then in Schiff's reagent for $40 \mathrm{~min}$, and finally in 5\% (w/v) sodium bisulfite for $35 \mathrm{~min}$. The sections were then rinsed in distilled water, dried on a hotplate, and mounted in Histomount. A negative control was prepared in the same manner, except the periodic acid oxidation step was omitted. The thin sections were observed under a light microscope (Axioscop 2; Carl Zeiss AG, Oberkochen, Germany).

Scanning electron microscopy (SEM): live tissues were examined under a SEM (SU-3500; Hitachi, Tokyo, Japan) in low vacuum mode.

\subsection{Statistical Analysis}

Data are means \pm standard error (SE). They were subjected to analysis of variance (ANOVA) and the level of significance was calculated from F value of ANOVA, following Duncan's multiple range test for each experiment at $p<0.05$. All data processing was performed in SAS v. 9.2 (SAS Institute, Cary, NC, USA).

\section{Results and Discussion}

\subsection{Fruit Quality}

The quality of the pepper fruit harvested at different times was determined during low-temperature storage. Fruit firmness increased with a delay in harvest time up to 45 DAF and decreased with an increase in storage period (Table 1). Firmness of the fruit harvested at 35-45 DAF was significantly higher than in fruit harvested at 15-30 DAF during cold storage at $4{ }^{\circ} \mathrm{C}$ for $15 \mathrm{~d}$ even after transfer to $20^{\circ} \mathrm{C}$. Soluble solid content (SSC) significantly increased with an increase in storage period. Similarly, TSS content in bell pepper increased gradually with storage periods [25]. Maximum SSC was observed in the fruit harvested at 50 DAF. The titratable acidity (TA) was higher in the fruit harvested at 35-50 DAF than in fruit harvested earlier. In general, TA reduction is a major characteristic feature during storage periods [26]. Consistently, pepper fruit acidity increased with harvest time showing fruit harvested at 50 DAF had the highest TA while fruit acidity decreased over the storage periods. The fruit harvested at a later stage (35 DAF to 50 DAF) exhibited higher firmness, SSC, and TA than those harvested at $15 \mathrm{DAF}$. Fruit hardness and flavor improve with post-flowering maturation. There were no significant differences in the color A value (redness) among fruits harvested before 35 DAF. Fruit color changed to red when fruits were transferred to shelf condition at $20^{\circ} \mathrm{C}$, after low-temperature storage (Figure 1B). Hence, 35 DAF may be the optimal harvest time required to obtain the fruit color and robustness preferred by consumers.

\subsection{Harvest Time and Seed Browning}

Peppers were harvested at 15, 20, 30, 35, 40, 45, and 50 DAF. Photographs showing the colors of the inner and outer sections of the fruits were taken during low-temperature storage $\left(4^{\circ} \mathrm{C}\right)$ and after transfer to shelf conditions $\left(20^{\circ} \mathrm{C}\right)$ (Figure 1A). The seeds of the fruit harvested $15 \mathrm{DAF}$ showed $100 \%$ browning within $7 \mathrm{~d}$ of low-temperature storage (CI index, 4.0$)$. In contrast, only $10 \%$ of the peppers harvested $35 \mathrm{DAF}$ displayed brown seeds (CI index, 0.4) (Figure 1C). There was no significant difference in $\mathrm{CI}$ index between $35 \mathrm{DAF}$ and $50 \mathrm{DAF}$ seeds following low-temperature storage $\left(4^{\circ} \mathrm{C}\right)$, and after transfer to shelf conditions $\left(20^{\circ} \mathrm{C}\right)$. Similarly, seed browning in hot pepper was severe in fruit stored at $5{ }^{\circ} \mathrm{C}$, but only in fruit harvested $15 \mathrm{DAF}$ [5]. In the present study, seed browning rates in the chilled samples were negatively related with harvest time (Figure 1). The CI index in seeds of fruits harvested from $15 \mathrm{DAF}$ to $30 \mathrm{DAF}$ was significantly higher than in fruits harvested from $35 \mathrm{DAF}$ to $50 \mathrm{DAF}$ when fruits were stored for 15 days at $4{ }^{\circ} \mathrm{C}$ and 4 days at $20^{\circ} \mathrm{C}$ (Figure 1C). In addition, pepper fruit harvested from $15 \mathrm{DAF}$ to $30 \mathrm{DAF}$ exhibited seed browning without ripening progress until 15 days of cold storage (Figure 1A), which is consistent with the finding of a previous study in which pepper fruit stored at $2{ }^{\circ} \mathrm{C}$ exhibited seed browning without ripening progress for 25 days [10]. Therefore, the results suggest that harvesting time of hot pepper "Nockgwang" significantly influences 
seed browning, and harvesting pepper after 35 DAF can suppress seed browning under long-term low-temperature storage $\left(4^{\circ} \mathrm{C}\right)$.

Table 1. Changes in pepper fruit firmness, soluble solid content (SSC), and titratable acidity (TA) during storage.

\begin{tabular}{|c|c|c|c|c|}
\hline & 0 day & 7 days & 15 days & $15+4$ days \\
\hline & \multicolumn{4}{|c|}{ Firmness (N) } \\
\hline $15 \mathrm{DAF}$ & $9.63 \pm 0.34 \mathrm{Fa}$ & $10.46 \pm 0.32 \mathrm{Da}$ & $6.51 \pm 0.43 \mathrm{CDb}$ & $5.12 \pm 0.24 \mathrm{CDc}$ \\
\hline $20 \mathrm{DAF}$ & $11.05 \pm 0.37 \mathrm{Ea}$ & $10.63 \pm 0.25 \mathrm{Da}$ & $6.08 \pm 0.26 \mathrm{Db}$ & $4.85 \pm 0.20 \mathrm{Dc}$ \\
\hline $25 \mathrm{DAF}$ & $12.94 \pm 0.33 \mathrm{Da}$ & $12.62 \pm 0.39 \mathrm{Ca}$ & $7.28 \pm 0.24 \mathrm{Cb}$ & $5.35 \pm 0.26 \mathrm{CDc}$ \\
\hline $30 \mathrm{DAF}$ & $13.80 \pm 0.31 \mathrm{CDa}$ & $11.82 \pm 0.33 \mathrm{Cb}$ & $8.67 \pm 0.37 \mathrm{Bc}$ & $5.15 \pm 0.28 \mathrm{CDd}$ \\
\hline $35 \mathrm{DAF}$ & $15.76 \pm 0.40 \mathrm{Ba}$ & $11.94 \pm 0.43 \mathrm{Cb}$ & $9.23 \pm 0.28 \mathrm{Bc}$ & $6.55 \pm 0.25 \mathrm{Bd}$ \\
\hline $40 \mathrm{DAF}$ & $18.91 \pm 0.49 \mathrm{Aa}$ & $14.42 \pm 0.49 \mathrm{Bb}$ & $10.71 \pm 0.21 \mathrm{Ac}$ & $7.55 \pm 0.34 \mathrm{Ad}$ \\
\hline $45 \mathrm{DAF}$ & $18.16 \pm 0.43 \mathrm{Aa}$ & $15.97 \pm 0.44 \mathrm{Ab}$ & $11.20 \pm 0.25 \mathrm{Ac}$ & $7.94 \pm 0.38 \mathrm{Ad}$ \\
\hline \multirow[t]{2}{*}{$50 \mathrm{DAF}$} & $14.75 \pm 0.33 \mathrm{BCa}$ & $13.97 \pm 0.30 \mathrm{Bb}$ & $8.65 \pm 0.25 \mathrm{Bc}$ & $5.73 \pm 0.20 \mathrm{Cd}$ \\
\hline & \multicolumn{4}{|c|}{ Soluble solids content (\%) } \\
\hline $15 \mathrm{DAF}$ & $5.53 \pm 0.12 \mathrm{Dd}$ & $6.40 \pm 0.12 \mathrm{DEc}$ & $6.90 \pm 0.00 \mathrm{Cb}$ & $7.70 \pm 0.00 \mathrm{Da}$ \\
\hline $20 \mathrm{DAF}$ & $5.30 \pm 0.06 \mathrm{Ec}$ & $6.67 \pm 0.03 \mathrm{Dab}$ & $6.60 \pm 0.00 \mathrm{Cb}$ & $6.83 \pm 0.09 \mathrm{Ea}$ \\
\hline $25 \mathrm{DAF}$ & $5.30 \pm 0.00 \mathrm{Ed}$ & $6.07 \pm 0.13 \mathrm{EFc}$ & $6.60 \pm 0.06 \mathrm{Cb}$ & $7.17 \pm 0.15 \mathrm{DEa}$ \\
\hline $30 \mathrm{DAF}$ & $5.33 \pm 0.03 \mathrm{Ed}$ & $5.93 \pm 0.03 \mathrm{Fc}$ & $6.60 \pm 0.06 \mathrm{Cb}$ & $7.07 \pm 0.15 \mathrm{DEa}$ \\
\hline $35 \mathrm{DAF}$ & $5.37 \pm 0.03 \mathrm{DEc}$ & $6.20 \pm 0.17 \mathrm{EFb}$ & $6.50 \pm 0.06 \mathrm{Cb}$ & $6.97 \pm 0.18 \mathrm{Ea}$ \\
\hline $40 \mathrm{DAF}$ & $5.90 \pm 0.00 \mathrm{Cc}$ & $7.20 \pm 0.12 \mathrm{Cb}$ & $6.87 \pm 0.15 \mathrm{Cb}$ & $8.87 \pm 0.50 \mathrm{Ca}$ \\
\hline $45 \mathrm{DAF}$ & $7.33 \pm 0.07 \mathrm{Bc}$ & $8.53 \pm 0.15 \mathrm{Bb}$ & $9.60 \pm 0.12 \mathrm{Ba}$ & $9.80 \pm 0.25 \mathrm{Ba}$ \\
\hline \multirow[t]{2}{*}{$50 \mathrm{DAF}$} & $9.83 \pm 0.03 \mathrm{Ad}$ & $10.60 \pm 0.17 \mathrm{Ac}$ & $11.97 \pm 0.32 \mathrm{Ab}$ & $13.50 \pm 0.00 \mathrm{Aa}$ \\
\hline & \multicolumn{4}{|c|}{ Titratable acidity (\%) } \\
\hline $15 \mathrm{DAF}$ & $0.12 \pm 0.00 \mathrm{Ga}$ & $0.07 \pm 0.00 \mathrm{Fb}$ & $0.07 \pm 0.00 \mathrm{~Gb}$ & $0.07 \pm 0.00 \mathrm{Db}$ \\
\hline $20 \mathrm{DAF}$ & $0.13 \pm 0.00 \mathrm{~Gb}$ & $0.17 \pm 0.01 \mathrm{Ea}$ & $0.08 \pm 0.00 \mathrm{Fc}$ & $0.07 \pm 0.00 \mathrm{Dc}$ \\
\hline $25 \mathrm{DAF}$ & $0.21 \pm 0.00 \mathrm{Fa}$ & $0.21 \pm 0.01 \mathrm{Da}$ & $0.10 \pm 0.00 \mathrm{DEb}$ & $0.10 \pm 0.02 \mathrm{Cb}$ \\
\hline $30 \mathrm{DAF}$ & $0.25 \pm 0.02 \mathrm{Ea}$ & $0.23 \pm 0.01 \mathrm{Da}$ & $0.09 \pm 0.00 \mathrm{~Eb}$ & $0.12 \pm 0.02 \mathrm{Cb}$ \\
\hline $35 \mathrm{DAF}$ & $0.29 \pm 0.01 \mathrm{Da}$ & $0.25 \pm 0.00 \mathrm{Db}$ & $0.10 \pm 0.00 \mathrm{Dc}$ & $0.11 \pm 0.01 \mathrm{Cc}$ \\
\hline $40 \mathrm{DAF}$ & $0.34 \pm 0.00 \mathrm{Ca}$ & $0.34 \pm 0.01 \mathrm{Ca}$ & $0.11 \pm 0.00 \mathrm{Cc}$ & $0.20 \pm 0.01 \mathrm{Bb}$ \\
\hline $45 \mathrm{DAF}$ & $0.45 \pm 0.01 \mathrm{Ba}$ & $0.45 \pm 0.01 \mathrm{Ba}$ & $0.19 \pm 0.00 \mathrm{Bc}$ & $0.23 \pm 0.00 \mathrm{ABb}$ \\
\hline $50 \mathrm{DAF}$ & $0.52 \pm 0.03 \mathrm{Aa}$ & $0.54 \pm 0.01 \mathrm{Aa}$ & $0.22 \pm 0.00 \mathrm{Ab}$ & $0.24 \pm 0.00 \mathrm{Ab}$ \\
\hline
\end{tabular}

Fruits were harvested at $15,20,25,30,35,40,45$, and 50 days after flowering (DAF) and stored for 15 days at $4{ }^{\circ} \mathrm{C}$ followed by $4 \mathrm{~d}$ at $20^{\circ} \mathrm{C}(15+4$ days). Data are means \pm standard error (SE), $(\mathrm{n}=20$ for firmness; $\mathrm{n}=15$ for SSC and TA). Means with the same uppercase letter in a column or same lowercase letter in a row are not significantly different at $p<0.05$ according to Duncan's multiple range test.

\subsection{Antioxidant Capacity, Total Phenol Content}

Cell membranes are particularly susceptible to CI [27], as cold stress induces the generation of ROS associated with seed browning in hot pepper [9]. Here, we measured antioxidant activity in hot pepper seeds and pericarps at various developmental stages using the1,1-diphenyl-2-picrylhydrazyl (DPPH) and 2,2-azino-bis-3-ethylbenzothiazoline-6-sulfonic acid (ABTS) assays (Tables 2 and 3). Antioxidant activity was the highest in 15 DAF seeds, but steadily declined thereafter, until 50 DAF. The seeds in fruit harvested 15 DAF had high antioxidant content early in storage but relatively low antioxidant levels under shelf conditions following low-temperature storage (Table 2). Under shelf conditions $\left(20^{\circ} \mathrm{C}\right)$, after 15 days of low-temperature storage, the antioxidant levels detected based on DPPH were significantly higher 35 DAF than at other maturity stages (Table 2). The antioxidant activity in the seeds of fruit harvested 15 DAF was significantly higher than in seeds of fruit harvested 
40-50 DAF, under 7 days low-temperature storage, with the seeds in fruit harvested 15 DAF exhibiting $100 \%$ browning (CI index, 4.0). In contrast, the seeds in fruit harvested 40-50 DAF exhibited no browning (Figure 2). Therefore, the antioxidant activity in the seeds did not prevent browning entirely during low-temperature storage.

Table 2. Changes in 1,1-diphenyl-2-picrylhydrazyl (DPPH), 2,2-azino-bis-3-ethylbenzothiazoline-6sulfonic acid (ABTS), and total phenolic content in hot pepper seeds during storage.

\begin{tabular}{|c|c|c|c|c|}
\hline & 0 day & 7 days & 15 days & $15+4$ days \\
\hline & \multicolumn{4}{|c|}{ DPPH (Trolox equivalent, $\mu \mathrm{mol} / \mathrm{g}$ DW) } \\
\hline $15 \mathrm{DAF}$ & $5.19 \pm 0.07 \mathrm{Aa}$ & $4.79 \pm 0.10 \mathrm{Db}$ & $4.76 \pm 0.02 \mathrm{Cb}$ & $3.64 \pm 0.05 \mathrm{Cc}$ \\
\hline $20 \mathrm{DAF}$ & $5.13 \pm 0.08 \mathrm{Ab}$ & $5.42 \pm 0.03 \mathrm{Aa}$ & $5.20 \pm 0.02 \mathrm{Ab}$ & $3.18 \pm 0.04 \mathrm{Dc}$ \\
\hline $25 \mathrm{DAF}$ & $4.59 \pm 0.09 \mathrm{Cb}$ & $5.01 \pm 0.02 \mathrm{Ca}$ & $4.94 \pm 0.04 \mathrm{Ba}$ & $3.73 \pm 0.02 \mathrm{Cc}_{\mathrm{c}}$ \\
\hline $30 \mathrm{DAF}$ & $4.60 \pm 0.06 \mathrm{Cc}$ & $5.23 \pm 0.02 \mathrm{Ba}$ & $4.94 \pm 0.02 \mathrm{Bb}$ & $3.90 \pm 0.03 \mathrm{Bd}$ \\
\hline $35 \mathrm{DAF}$ & $4.93 \pm 0.04 \mathrm{Bb}$ & $5.08 \pm 0.03 \mathrm{Ca}$ & $4.85 \pm 0.03 \mathrm{BCb}$ & $4.18 \pm 0.07 \mathrm{Ac}$ \\
\hline $40 \mathrm{DAF}$ & $3.88 \pm 0.02 \mathrm{~Eb}$ & $4.44 \pm 0.07 \mathrm{Ea}$ & $4.44 \pm 0.06 \mathrm{Da}$ & $3.98 \pm 0.03 \mathrm{Bb}$ \\
\hline $45 \mathrm{DAF}$ & $4.36 \pm 0.04 \mathrm{Da}$ & $4.03 \pm 0.03 \mathrm{Fc}$ & $4.20 \pm 0.03 \mathrm{~Eb}$ & $3.90 \pm 0.06 \mathrm{Bc}$ \\
\hline \multirow[t]{2}{*}{$50 \mathrm{DAF}$} & $3.89 \pm 0.05 \mathrm{~Eb}$ & $4.04 \pm 0.04 \mathrm{Fa}$ & $3.93 \pm 0.04 \mathrm{Fab}$ & $3.99 \pm 0.04 \mathrm{Bab}$ \\
\hline & \multicolumn{4}{|c|}{ ABTS (Trolox equivalent, $\mu \mathrm{mol} / \mathrm{g}$ DW) } \\
\hline $15 \mathrm{DAF}$ & $15.78 \pm 1.09 \mathrm{ABa}$ & $15.65 \pm 1.05 \mathrm{Ca}$ & $13.33 \pm 0.72 \mathrm{CDa}$ & $14.02 \pm 0.91 \mathrm{ABa}$ \\
\hline $20 \mathrm{DAF}$ & $17.40 \pm 1.15 \mathrm{Ab}$ & $21.16 \pm 0.61 \mathrm{Aa}$ & $17.50 \pm 0.73 \mathrm{Ab}$ & $12.13 \pm 0.67 \mathrm{Cc}$ \\
\hline $25 \mathrm{DAF}$ & $14.69 \pm 0.95 \mathrm{Bb}$ & $17.60 \pm 0.36 \mathrm{Ba}$ & $17.12 \pm 0.50 \mathrm{ABa}$ & $14.71 \pm 0.34 \mathrm{Ab}$ \\
\hline $30 \mathrm{DAF}$ & $16.96 \pm 0.58 \mathrm{ABab}$ & $18.39 \pm 0.73 \mathrm{Ba}$ & $16.35 \pm 0.57 \mathrm{ABb}$ & $14.08 \pm 0.49 \mathrm{ABC}$ \\
\hline $35 \mathrm{DAF}$ & $14.97 \pm 0.78 \mathrm{ABa}$ & $15.58 \pm 0.72 \mathrm{Ca}$ & $15.18 \pm 0.74 \mathrm{BCa}$ & $12.77 \pm 0.53 \mathrm{BCb}$ \\
\hline $40 \mathrm{DAF}$ & $7.33 \pm 0.43 \mathrm{Db}$ & $11.11 \pm 0.46 \mathrm{Da}$ & $11.62 \pm 1.00 \mathrm{Da}$ & $8.50 \pm 0.60 \mathrm{Db}$ \\
\hline $45 \mathrm{DAF}$ & $10.94 \pm 0.58 \mathrm{Ca}$ & $9.53 \pm 0.47 \mathrm{Dab}$ & $8.06 \pm 0.57 \mathrm{~Eb}$ & $7.89 \pm 0.63 \mathrm{Db}$ \\
\hline \multirow[t]{2}{*}{$50 \mathrm{DAF}$} & $8.55 \pm 0.62 \mathrm{Dab}$ & $9.29 \pm 0.47 \mathrm{Da}$ & $7.29 \pm 0.62 \mathrm{~Eb}$ & $8.69 \pm 0.64 \mathrm{Dab}$ \\
\hline & \multicolumn{4}{|c|}{ Total phenolics (gallic acid equivalent, mg/g DW) } \\
\hline $15 \mathrm{DAF}$ & $4.66 \pm 0.01 \mathrm{Aa}$ & $4.57 \pm 0.00 \mathrm{Ab}$ & $4.53 \pm 0.00 \mathrm{Ac}$ & $3.33 \pm 0.00 \mathrm{Ad}$ \\
\hline $20 \mathrm{DAF}$ & $3.67 \pm 0.02 \mathrm{Bb}$ & $4.01 \pm 0.01 \mathrm{Ba}$ & $4.04 \pm 0.04 \mathrm{Ba}$ & $2.66 \pm 0.01 \mathrm{Bc}$ \\
\hline $25 \mathrm{DAF}$ & $2.65 \pm 0.02 \mathrm{Db}$ & $2.56 \pm 0.01 \mathrm{Cc}$ & $3.41 \pm 0.01 \mathrm{Ca}$ & $2.54 \pm 0.01 \mathrm{Cc}$ \\
\hline $30 \mathrm{DAF}$ & $2.73 \pm 0.02 \mathrm{Ca}$ & $2.44 \pm 0.00 \mathrm{Db}$ & $2.46 \pm 0.01 \mathrm{Db}$ & $2.20 \pm 0.00 \mathrm{Dc}$ \\
\hline 35 DAF & $1.88 \pm 0.02 \mathrm{Ec}$ & $2.22 \pm 0.01 \mathrm{~Eb}$ & $2.29 \pm 0.01 \mathrm{Ea}$ & $1.86 \pm 0.01 \mathrm{Ec}$ \\
\hline $40 \mathrm{DAF}$ & $1.11 \pm 0.01 \mathrm{Gd}$ & $1.52 \pm 0.01 \mathrm{Fb}$ & $1.66 \pm 0.01 \mathrm{Fa}$ & $1.31 \pm 0.01 \mathrm{FC}_{\mathrm{C}}$ \\
\hline $45 \mathrm{DAF}$ & $1.54 \pm 0.01 \mathrm{Fa}$ & $1.20 \pm 0.01 \mathrm{~Gb}$ & $1.15 \pm 0.00 \mathrm{Gc}$ & $1.21 \pm 0.01 \mathrm{~Gb}$ \\
\hline $50 \mathrm{DAF}$ & $1.12 \pm 0.01 \mathrm{Ga}$ & $1.10 \pm 0.02 \mathrm{Hab}$ & $1.12 \pm 0.01 \mathrm{Ga}$ & $1.08 \pm 0.01 \mathrm{Hb}$ \\
\hline
\end{tabular}

Fruits were harvested $15,20,25,30,35,40,45$, and 50 days after flowering (DAF) and stored for 15 days at $4{ }^{\circ} \mathrm{C}$ and then for 4 days at $20^{\circ} \mathrm{C}(15+4$ days). Means with the same uppercase letter in a column or same lowercase letter in a row are not significantly different at $p<0.05$ according to Duncan's multiple range test. 
Table 3. Changes in 1,1-diphenyl-2-picrylhydrazyl (DPPH), 2,2-azino-bis-3-ethylbenzothiazoline-6sulfonic acid (ABTS), and total phenolic content in pepper pericarp during storage.

\begin{tabular}{|c|c|c|c|c|}
\hline & 0 day & 7 days & 15 days & $15+4$ days \\
\hline & \multicolumn{4}{|c|}{ DPPH (Trolox equivalent, $\mu \mathrm{mol} / \mathrm{g}$ DW) } \\
\hline $15 \mathrm{DAF}$ & $28.74 \pm 1.21 \mathrm{Da}$ & $26.05 \pm 0.68 \mathrm{Cb}$ & $20.08 \pm 1.00 \mathrm{Ec}$ & $29.17 \pm 0.52 \mathrm{Da}$ \\
\hline $20 \mathrm{DAF}$ & $35.96 \pm 1.16 \mathrm{BCa}$ & $27.01 \pm 0.39 \mathrm{Cb}$ & $29.70 \pm 1.79 \mathrm{Cb}$ & $28.03 \pm 0.42 \mathrm{Db}$ \\
\hline $25 \mathrm{DAF}$ & $34.43 \pm 0.29 \mathrm{Ca}$ & $28.23 \pm 0.87 \mathrm{BCb}$ & $28.23 \pm 0.95 \mathrm{Cb}$ & $36.06 \pm 0.54 \mathrm{Ba}$ \\
\hline $30 \mathrm{DAF}$ & $24.89 \pm 0.33 \mathrm{Ec}$ & $27.58 \pm 0.46 \mathrm{BCb}$ & $24.68 \pm 0.35 \mathrm{Dc}$ & $32.58 \pm 0.95 \mathrm{Ca}$ \\
\hline $35 \mathrm{DAF}$ & $23.67 \pm 0.56 \mathrm{Ec}$ & $26.97 \pm 0.43 \mathrm{Cb}$ & $22.24 \pm 0.31 \mathrm{DEc}$ & $31.65 \pm 0.77 \mathrm{Ca}$ \\
\hline $40 \mathrm{DAF}$ & $27.72 \pm 0.98 \mathrm{Da}$ & $22.13 \pm 0.94 \mathrm{Db}$ & $27.93 \pm 0.65 \mathrm{Ca}$ & $29.40 \pm 0.34 \mathrm{Da}$ \\
\hline $45 \mathrm{DAF}$ & $37.09 \pm 0.49 \mathrm{Bab}$ & $30.27 \pm 1.60 \mathrm{Bc}$ & $38.02 \pm 0.42 \mathrm{Ba}$ & $35.30 \pm 0.50 \mathrm{Bb}$ \\
\hline \multirow[t]{2}{*}{$50 \mathrm{DAF}$} & $42.64 \pm 0.81 \mathrm{Ab}$ & $39.99 \pm 1.29 \mathrm{Ac}$ & $47.83 \pm 0.51 \mathrm{Aa}$ & $40.61 \pm 0.68 \mathrm{Abc}$ \\
\hline & \multicolumn{4}{|c|}{ ABTS (Trolox equivalent, $\mu \mathrm{mol} / \mathrm{g}$ DW) } \\
\hline $15 \mathrm{DAF}$ & $42.49 \pm 1.44 \mathrm{Bb}$ & $30.51 \pm 0.49 \mathrm{Gc}$ & $27.62 \pm 0.19 \mathrm{Fd}$ & $45.44 \pm 0.53 \mathrm{Ca}$ \\
\hline $20 \mathrm{DAF}$ & $45.40 \pm 1.47 \mathrm{Ba}$ & $38.69 \pm 0.11 \mathrm{~Eb}$ & $45.98 \pm 0.54 \mathrm{Ca}$ & $44.29 \pm 0.30 \mathrm{Ca}$ \\
\hline $25 \mathrm{DAF}$ & $35.67 \pm 1.53 \mathrm{Cc}$ & $45.04 \pm 0.52 \mathrm{Cb}$ & $46.49 \pm 0.26 \mathrm{Cb}$ & $57.53 \pm 0.12 \mathrm{Aa}$ \\
\hline $30 \mathrm{DAF}$ & $33.58 \pm 0.61 \mathrm{Cd}$ & $46.13 \pm 0.24 \mathrm{Bb}$ & $37.20 \pm 0.57 \mathrm{Ec}$ & $52.33 \pm 0.35 \mathrm{Ba}$ \\
\hline $35 \mathrm{DAF}$ & $34.02 \pm 1.96 \mathrm{Cc}$ & $38.38 \pm 0.13 \mathrm{~Eb}$ & $36.31 \pm 0.33 \mathrm{Ebc}$ & $44.07 \pm 0.56 \mathrm{Ca}$ \\
\hline $40 \mathrm{DAF}$ & $34.98 \pm 1.76 \mathrm{Cb}$ & $34.24 \pm 0.18 \mathrm{Fb}$ & $41.89 \pm 0.50 \mathrm{Da}$ & $40.78 \pm 0.52 \mathrm{Da}$ \\
\hline $45 \mathrm{DAF}$ & $42.87 \pm 0.83 \mathrm{Bb}$ & $41.69 \pm 0.39 \mathrm{Db}$ & $51.22 \pm 0.32 \mathrm{Ba}$ & $50.87 \pm 0.89 \mathrm{Ba}$ \\
\hline \multirow[t]{2}{*}{$50 \mathrm{DAF}$} & $50.53 \pm 0.81 \mathrm{Ab}$ & $50.58 \pm 0.34 \mathrm{Ab}$ & $55.76 \pm 0.11 \mathrm{Aa}$ & $51.80 \pm 0.65 \mathrm{Bb}$ \\
\hline & \multicolumn{4}{|c|}{ Total phenol (gallic acid equivalent, mg/g DW) } \\
\hline $15 \mathrm{DAF}$ & $3.71 \pm 0.13 \mathrm{Cab}$ & $3.46 \pm 0.01 \mathrm{~Eb}$ & $3.70 \pm 0.12 \mathrm{Dab}$ & $3.82 \pm 0.03 \mathrm{Da}$ \\
\hline $20 \mathrm{DAF}$ & $3.91 \pm 0.05 \mathrm{Ba}$ & $3.53 \pm 0.01 \mathrm{Dec}$ & $3.76 \pm 0.01 \mathrm{Db}$ & $3.42 \pm 0.02 \mathrm{Fd}$ \\
\hline $25 \mathrm{DAF}$ & $3.71 \pm 0.01 \mathrm{Cc}$ & $3.79 \pm 0.02 \mathrm{Cb}$ & $3.82 \pm 0.01 \mathrm{Db}$ & $4.42 \pm 0.02 \mathrm{Aa}$ \\
\hline $30 \mathrm{DAF}$ & $3.23 \pm 0.02 \mathrm{Dd}$ & $4.00 \pm 0.05 \mathrm{Ab}$ & $3.46 \pm 0.00 \mathrm{Ec}$ & $4.24 \pm 0.01 \mathrm{Ba}$ \\
\hline $35 \mathrm{DAF}$ & $3.31 \pm 0.01 \mathrm{Dc}$ & $3.59 \pm 0.02 \mathrm{Db}$ & $3.13 \pm 0.02 \mathrm{Fd}$ & $3.88 \pm 0.02 \mathrm{Ca}$ \\
\hline $40 \mathrm{DAF}$ & $3.37 \pm 0.01 \mathrm{Dc}$ & $3.39 \pm 0.02 \mathrm{Fc}$ & $4.18 \pm 0.02 \mathrm{Ca}$ & $3.65 \pm 0.01 \mathrm{~Eb}$ \\
\hline $45 \mathrm{DAF}$ & $3.67 \pm 0.06 \mathrm{Cd}$ & $3.93 \pm 0.01 \mathrm{Bc}$ & $4.39 \pm 0.01 \mathrm{Ba}$ & $4.27 \pm 0.02 \mathrm{Bb}$ \\
\hline $50 \mathrm{DAF}$ & $4.19 \pm 0.01 \mathrm{Ac}$ & $4.06 \pm 0.02 \mathrm{Ad}$ & $4.75 \pm 0.02 \mathrm{Aa}$ & $4.41 \pm 0.00 \mathrm{Ab}$ \\
\hline
\end{tabular}

Fruits were harvested at $15,20,25,30,35,40,45$, and 50 days after flowering (DAF) and stored for 15 days at $4{ }^{\circ} \mathrm{C}$ and for 4 days at $20^{\circ} \mathrm{C}(15+4$ days). Means with the same uppercase letter in a column or same lowercase letter in a row were not significantly different at $p<0.05$ according to Duncan's multiple range test.

Antioxidant activity is positively correlated with vegetable freshness. Oxidative stress responses are associated with the emergence of CI symptoms such as pepper fruit pitting [2]. Here, we compared antioxidant activity in the pericarp and seeds. The pericarp of fruits harvested 35-50 DAF exhibited relatively higher antioxidant activity than those of the pericarp of fruits harvested 15 DAF. Pericarp of fruit harvested 50 DAF had the highest ABTS and DPPH content (Table 3). The findings are consistent with those reported by Jang et al. [28], who demonstrated that antioxidant activity 43-48 days post-anthesis (DPA) was similar to those in earlier stages. The response could explain why the seeds did not brown during cold storage.

Tissue browning induced by $\mathrm{CI}$ is associated with polyphenol oxidase and phenylalanine ammonialyase production. Such enzymes participate in phenolic biosynthesis and polymerization [5,29]. The total phenolic levels in the seeds of fruit harvested 15 DAF were significantly higher than in those harvested at any other stage under shelf conditions. In other words, brown seeds in fruit harvested 15 DAF had comparatively higher total phenolic content than those of non-browning seeds in fruit harvested 50 DAF during low-temperature storage (Table 2). The above results suggest total 
phenolic levels might be involved in the degree of seed browning at different harvest times under low-temperature storage.
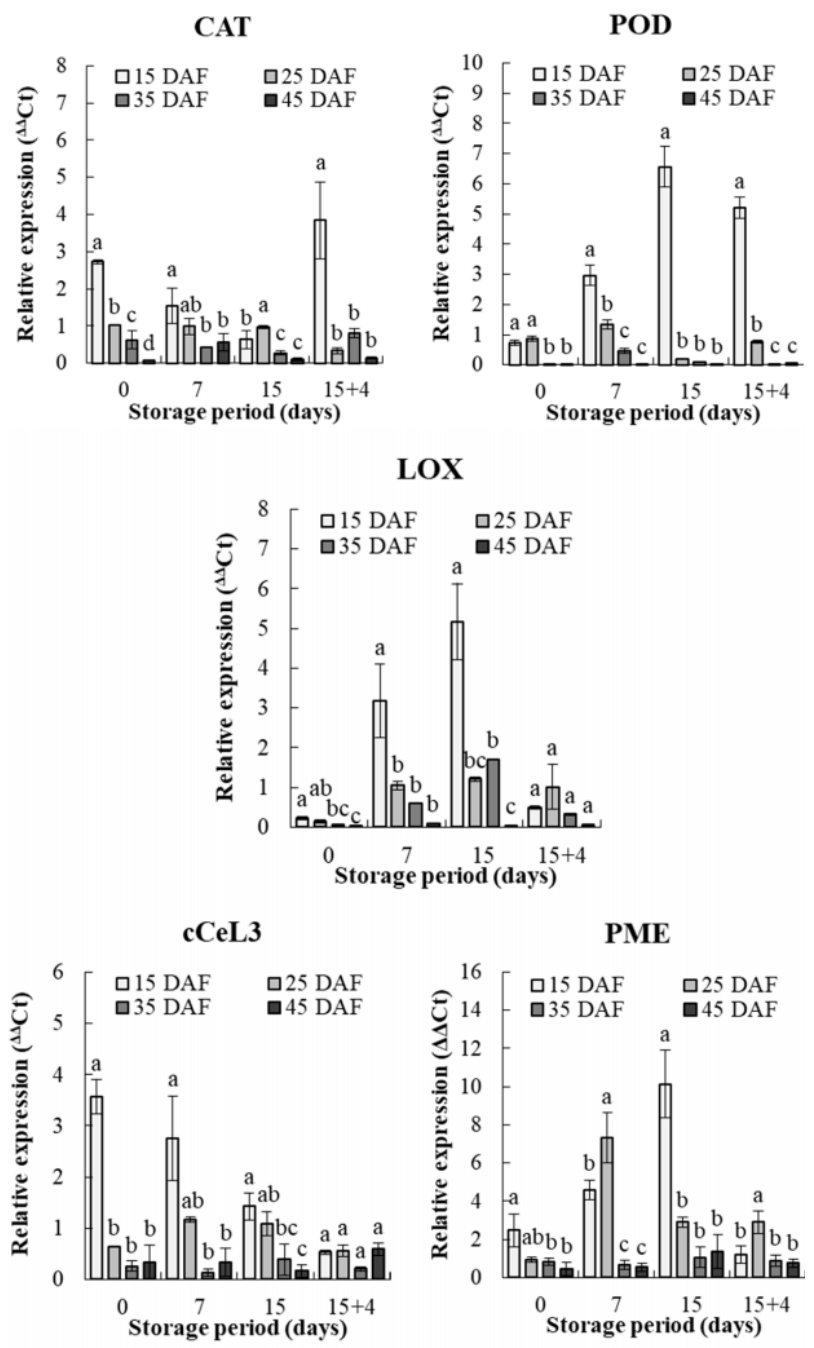

PBG1

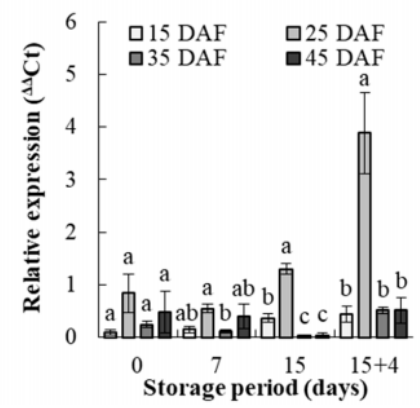

Figure 2. Quantitative real-time polymerase chain reaction (RT-qPCR) analysis of the transcript levels of catalase (CAT), lipoxygenase (LOX), and peroxidase (POX) (upper panel), endo- $\beta$-1,4-glucanase (cCeL3), pectin methylesterase-like protein (PME), and $\beta$-Galactosidase-1 (PBG1) in seeds of hot pepper. Data are means \pm SE of three replicates. Different letters indicate significant differences among treatments within the same storage time according to the Duncan's multiple range test $(p<0.05)$. Fruits were harvested $15,25,35$, and 45 days after flowering (DAF) and stored for 15 days at $4{ }^{\circ} \mathrm{C}$ and for 4 days at $20^{\circ} \mathrm{C}(15+4)$. 


\subsection{Analysis of the Expression Levels of Antioxidant-and Cell Wall-Related Genes During Storage}

To confirm the relationships observed between seed browning and antioxidant levels during cold storage, we investigated the levels of expression of antioxidant-related genes in seeds of hot pepper. Gene expression levels of catalase (CAT) and peroxidase (POD) were significantly upregulated in the seeds of fruit harvested $15 \mathrm{DAF}$ and $25 \mathrm{DAF}$, when compared to the levels in those harvested $35 \mathrm{DAF}$ and $45 \mathrm{DAF}$, under 7 days of cold storage (Figure 2). The findings corroborate those for antioxidant levels in the seeds evaluated using ABTS and DPPH assays (Table 2). However, according to Boonsiri et al. [5], CAT and POD activity in seeds of fruits harvested 25 DAF was significantly higher than in seeds from fruit harvested $15 \mathrm{DAF}$, suggesting a negative correlation between browning and CAT and POD activity. Lee et al. [9] showed that genes related to superoxide dismutase, CAT, and ascorbate peroxidase were significantly upregulated in hot pepper seeds at 20 days and $2{ }^{\circ} \mathrm{C}$, when seed browning was severe. The results of the present study revealed antioxidant gene expression was upregulated in immature seeds showing severe seed browning during cold storage.

Seed browning might be associated with cell membrane peroxidation by LOX [9]. In the present study, LOX expression in the seeds of fruit harvested 15 DAF was significantly higher than in seeds of fruit harvested later (35 DAF to $45 \mathrm{DAF}$ ) during low-temperature storage. Here, we confirmed that cold stress induced LOX, which caused membrane lipid peroxidation, disrupted membrane integrity, and induced seed browning under low-temperature storage. We also examined the expression of cell wall-related genes during pepper fruit storage. The expression of pepper endo- $\beta-1,4$-glucanase ( $c$ CeL3) was significantly upregulated in seeds harvested 15 DAF than in seeds of fruits harvested at later stages, during low-temperature storage. However, enzyme activity had the lowest initial value in fruits harvested $\sim 35$ DAF. Pepper $c C e L 3$ is involved in leaf abscission [30]. Endo-1,4- $\beta$-glucanase (EGase) is upregulated during fruit ripening and requires cooperative cell wall component disassembly during ripening and abscission [31]. The upregulation of $c C e L 3$ in the seeds of fruit harvested $15 \mathrm{DAF}$ might be associated with the softening of young seeds as EGase mediates fruit softening [32]. Seeds in fruit harvested 15 DAF were softer than those in fruit harvested 35 DAF and 45 DAF. PME1 expression in the seeds of fruit harvested 15 DAF and 25 DAF was significantly higher than in seeds of fruit harvested $35 \mathrm{DAF}$ and $45 \mathrm{DAF}$, during cold storage. PME hydrolyzes pectin, which is the primary constituent of the middle lamella and the primary cell wall. The expression of PME1 in the seeds of fruit harvested $15 \mathrm{DAF}$ and $25 \mathrm{DAF}$ increased under low-temperature storage (Figure 2). PME1 upregulation may cause cell wall softening. Pepper $\beta$-galactosidase-1 (PBG1) gene expression in seeds of fruit harvested 25 DAF was significantly higher than in 35 DAF and 45 DAF fruits, following 15 days of cold storage, and even after transfer to $20^{\circ} \mathrm{C}$. The young seeds, which are sensitive to cold stress and chilling, may initiate cell wall degradation. Previous study showed, using pericarp of bell pepper, PBG1 gene participates in fruit ripening [33]. Pectinesterase and $\beta$-galactosidase have been associated with CI sensitivity and loss of firmness in carambola fruit [34].

\subsection{Anatomical Analysis}

In the present study, we explored the relationship between hot pepper seed maturity and CI by examining cell wall integrity after cryopreservation of seeds harvested at various times. Stereomicroscope images of seeds of pepper fruit harvested 15, 25, 35, and 45 DAF are shown in Figure 3A. After 7 days cold storage, the seeds of fruit harvested 15 DAF and 25 DAF browned. In contrast, the seeds of fruit harvested 35 DAF and 45 DAF exhibited no browning. We used SEM and LM to observe the structures of seeds obtained from fruits harvested at various times (Figure 3). The SEM images showed that the seed coat did not separate from the endosperm in the seeds of fruit harvested 25 DAF. These seeds exhibited browning after 7 days of storage at $4{ }^{\circ} \mathrm{C}$. In contrast, seeds from fruit harvested 35 DAF showed no browning; however, their seed coats were separated from their endosperms. The LM images revealed that the seeds from fruit harvested 35 DAF had fully developed seed coats and embryos. However, seeds from fruit harvested 25 DAF had damaged cells, only partially formed seed coats, and exhibited high sensitivity to cold storage. An earlier study 
reported that the early stages of pepper seed development were highly sensitive to high temperatures after anthesis. Hence, heat stress may have a detrimental effect on embryo formation [35].

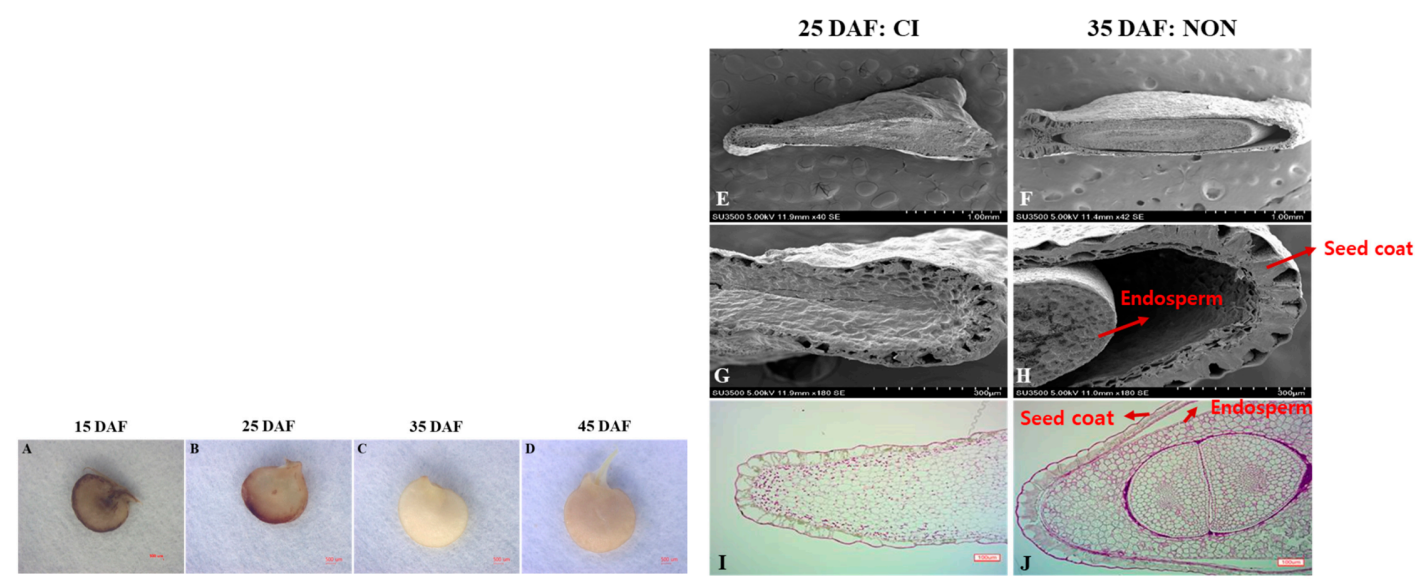

Figure 3. Pepper seed anatomy. (A-D). Stereomicroscope image of pepper seeds. Fruits were harvested $15,25,35$, and 45 days after flowering (DAF) and stored for 7 days at $4{ }^{\circ} \mathrm{C}$. (E-H) Scanning electron microscope (SEM) images and (I-J) light microscope (LM) images of pepper seed. Left: browning of seed in fruit harvested 25 DAF. Right: normal seed in fruit harvested 35 DAF. Fruits were harvested $25 \mathrm{DAF}$ or $35 \mathrm{DAF}$ and stored for 7 days at $4{ }^{\circ} \mathrm{C}$.

The results of the present study indicate that the degree of pepper seed browning varies with harvest time after flowering and is associated with changes in seed physicochemical properties. Seeds from fruit harvested after 35 DAF did not undergo browning. The seed coat may harden as the seeds mature after flowering. This change may confer physical resistance to cold stress. The above finding is consistent with the observation that chilled pepper fruit display visible symptoms of cell damage [14]. Similarly, in pea, the seed coat confers protection against CI [36].

\section{Conclusions}

The present study investigated the optimal harvest time that would minimize hot pepper seed browning induced by low-temperature storage. Harvesting pepper fruit 35 DAF resulted in excellent fruit quality and prevented seed browning. In contrast, the seeds of fruits harvested at 15 DAF had relatively higher antioxidant levels than those harvested later. Nevertheless, $100 \%$ of these seeds had turned brown within 7 days of cold storage. Cell wall degradation-related genes were upregulated and the seed coat was not fully developed until 25 DAF. Consequently, before seed maturity, the seeds were highly sensitive to chilling stress. However, the seeds of fruits harvested 35 DAF had fully formed seed coats. The primary reason why the seeds of fruits harvested 35 DAF did not turn brown during cold storage was that a mature hard seed coat conferred resistance to cold stress. This study confirmed that seed maturity in pepper was negatively correlated with seed browning. Therefore, antioxidant activities in seeds are not responsible for the browning that occurs in the seeds of immature pepper fruit subjected to low-temperature storage. The results of the present study suggest that producers could minimize postharvest losses in hot pepper production by harvesting fruits later than $35 \mathrm{DAF}$ that occur during long-term storage under low temperatures, and could facilitate the maintenance of the quality of hot pepper over longer periods, particularly in the course of long-distance export activities that require low-temperature distribution.

Supplementary Materials: The following are available online at http://www.mdpi.com/2077-0472/10/12/585/s1. Table S1. Primers used in the study of gene expression analysis.

Author Contributions: E.-Y.Y., J.-S.L., G.-R.D., and M.-H.P., experimental data and methodology; M.-H.P., Y.-P.H., original draft preparation, supervision, project administration, and funding acquisition; and M.-H.P., writing - review and editing, visualization, and data curation. All authors have read and agreed to the published version of the manuscript. 
Funding: This work was supported by Republic of Korea-National Institute of horticulture and Herbal Science, Rural Development Administration project (Project No. PJ01388901).

Conflicts of Interest: The authors declare no conflict of interest.

\section{References}

1. El-Ramady, H.R.; Domokos-Szabolcsy, É.; Abdalla, N.A.; Taha, H.S.; Fári, M. Postharvest management of fruit and vegetables storage. Sustain. Agric. Rev. 2015, 15, 65-152.

2. Shin, S.Y.; Park, M.H.; Choi, J.W.; Kim, J.G. Gene network underlying the response of harvested pepper to chilling stress. J. Plant Physiol. 2017, 219, 112-122. [CrossRef] [PubMed]

3. Özden, Ç.; Bayindirli, L. Effects of combinational use of controlled atmosphere, cold storage and edible coating applications on shelf life and quality attributes of green peppers. Eur. Food Res. Technol. 2002, 214, 320-326. [CrossRef]

4. Lim, C.S.; Woolf, A.B. Varietal differences of chilling-induced physiological responses and quality attributes in pepper (Capsicum annuum L.) cultivars during low temperature storage. Hortic. Environ. Biotechnol. 2010, 51, 531-538.

5. Boonsiri, K.; Ketsay, S.; Van Doorn, W.G. Seed browning of hot peppers during low temperature storage. Postharvest Biol. Technol. 2007, 45, 358-365. [CrossRef]

6. Lim, C.S.; Kang, S.M.; Cho, J.L.; Gross, K.C. Antioxidizing enzyme activities in chilling-sensitive and chilling-tolerant pepper fruit as affected by stage of ripeness and storage temperature. J. Am. Soc. Hortic. Sci. 2009, 134, 156-163. [CrossRef]

7. Wang, Q.; Ding, T.; Gao, L.; Pang, J.; Yang, N. Effect of brassinolide on chilling injury of green bell pepper in storage. Sci. Hortic. 2012, 144, 195-200. [CrossRef]

8. Wang, F.; Guo, Z.; Li, H.; Wang, M.; Onac, E.; Zhou, J.; Zhou, Y. Phytochrome A and B function antagonistically to regulate cold tolerance via abscisic acid-dependent jasmonate signaling. Plant Physiol. 2016, 170, 459-471. [CrossRef]

9. Lee, J.G.; Yi, G.; Choi, J.H.; Lee, E.J. Analyses of targeted/untargeted metabolites and reactive oxygen species of pepper fruits provide insights into seed browning induced by chilling. Food Chem. 2020, 332, 127406. [CrossRef]

10. Seo, J.; Yi, G.; Lee, J.G.; Choi, J.H.; Lee, E.J. Seed browning in pepper (Capsicum annuum L.) fruit during cold storage is inhibited by methyl jasmonate or induced by methyl salicylate. Postharvest Biol. Technol. 2020, 166, 111210. [CrossRef]

11. Lurie, S.; Watkins, C.B. Superficial scald, its etiology and control. Postharvest Biol. Technol. 2012, 65, 44-60. [CrossRef]

12. Vega-Garcia, M.O.; Lopez-Espinoza, G.; Ontiveros, J.C.; Caro-Corrales, J.J.; Vargas, F.D.; Lopez-Valenzuela, J.A. Changes in protein expression associated with chilling injury in tomato fruit. J. Am. Soc. Hortic. Sci. 2010, 135, 83-89. [CrossRef]

13. Saltveit, M.E. Fruit ripening and fruit quality. Crop Prod. Sci. Hortic. 2005, 13, 145.

14. Yang, J.; Fu, M.R.; Zhao, Y.Y.; Mao, L.C. Reduction of chilling injury and ultrastructural damage in cherry tomato fruits after hot water treatment. Agric. Sci. China 2009, 8, 304-310. [CrossRef]

15. Park, M.H.; Sangwanangkul, P.; Choi, J.W. Reduced chilling injury and delayed fruit ripening in tomatoes with modified atmosphere and humidity packaging. Sci. Hortic. 2018, 231, 66-72. [CrossRef]

16. Lin, W.C.; Hall, J.W.; Saltveit, M.E. Ripening stage affects the chilling sensitivity of greenhouse grown peppers. J. Am. Soc. Hortic. Sci. 1993, 118, 791-795. [CrossRef]

17. Abe, K.; Chachin, K.; Ogata, K. Chilling injury in eggplant fruits. II. The effects of maturation and harvesting season on pitting injury and browning of seeds and pulp during storage. J. Jpn. Soc. Hort. Sci. 1976, 45, 307-312. [CrossRef]

18. Sanchez-Bel, P.; Egea, I.; Sanchez-Ballesta, M.T.; Martinez-Madrid, C.; Fernandez-Garcia, N.; Romojaro, F.; Olmos, E.; Estrella, E.; Bolarín, M.C.; Flores, F.B. Understanding the mechanisms of chilling injury in bell pepper fruits using the proteomic approach. J. Proteom. 2012, 75, 5463-5478. [CrossRef]

19. Cao, S.; Zheng, Y.; Wang, K.; Jin, P.; Rui, H. Methyl jasmonate reduces chilling injury and enhances antioxidant enzyme activity in postharvest loquat fruit. Food Chem. 2009, 115, 1458-1463. [CrossRef] 
20. Singleton, V.L.; Rossi, J.A. Colorimetry of total phenolics with phosphomolybdic-phosphotungstic acid reagents. Am. J. Enol. Vitic. 1965, 16, 144-158.

21. Dietz, B.M.; Kang, Y.H.; Liu, G.; Eggler, A.L.; Yao, P.; Chadwick, L.R.; Pauli, G.F.; Farnsworth, N.R.; Mesecar, A.D.; Van Breemen, R.B.; et al. Xanthohumol isolated from Humulus lupulus inhibits menadione-induced DNA damage through induction of quinone reductase. Chem. Res. Toxicol. 2005, 18, 1296-1305. [CrossRef] [PubMed]

22. Re, R.; Pellegrigrini, N.; Proteggente, A.; Pannala, A.; Yang, M.; Rice-Evans, C. Antioxidant activity applying an improved ABTS radical cation decolorization assay. Free Radic. Biol. Med. 1999, 26, 1231-1237. [CrossRef]

23. Livak, K.J.; Schimittgen, T.D. Analysis of relative gene expression data using realtime quantitative PCR and the $2^{-\Delta \Delta}$ CT method. Methods 2001, 25, 402-408. [CrossRef] [PubMed]

24. Clément, C.; Burrus, M.; Audran, J.C. Floral organ growth and carbohydrate content during pollen development in Lilium. Am. J. Bot. 1996, 83, 459-469. [CrossRef]

25. Rao, T.R.; Gol, N.B.; Shah, K.K. Effect of postharvest treatments and storage temperatures on the quality and shelf life of sweet pepper (Capsicum annum L.). Sci. Hortic. 2011, 132, 18-26. [CrossRef]

26. Panigrahi, J.; Patel, M.; Patel, N.; Gheewala, B.; Gantait, S. Changes in antioxidant and biochemical activities in castor oil-coated Capsicum annuum L. during postharvest storage. 3 Biotech 2018, 8, 280. [CrossRef]

27. Shewfelt, R.L.; Del Rosario, B.A. The role of lipid peroxidation in storage disorders of fresh fruits and vegetables. HortScience 2000, 35, 575-579. [CrossRef]

28. Jang, Y.K.; Jung, E.S.; Lee, H.A.; Choi, D.; Lee, C.H. Metabolomic characterization of hot pepper (Capsicum annuum "CM334") during fruit development. J. Agric. Food Chem. 2015, 63, 9452-9460. [CrossRef]

29. Vamos Vigyazo, L.; Haard, N.F. Polyphenol oxidases and peroxidases in fruits and vegetables. Crit. Rev. Food Sci. Nutr. 1981, 15, 49-127. [CrossRef]

30. Trainotti, L.; Ferrarese, L.; Poznanski, E.; Dalla Vecchia, F. Endo- $\beta$-1, 4-glucanase activity is involved in the abscission of pepper flowers. J. Plant Physiol. 1998, 152, 70-77. [CrossRef]

31. Lashbrook, C.C.; Gonzalez-Bosch, C.; Bennett, A.B. Two divergent endo-beta-1, 4-glucanase genes exhibit overlapping expression in ripening fruit and abscising flowers. Plant Cell 1994, 6, 1485-1493. [CrossRef] [PubMed]

32. Roberts, J.A.; Gonzalez-Carranza, Z. Plant Cell Separation and Adhesion; Blackwell: Oxford, UK, 2007.

33. Ogasawara, S.; Abe, K.; Nakajima, T. Pepper beta-galactosidase 1 (PBG1) plays a significant role in fruit ripening in bell pepper (Capsicum annuum). Biosci. Biotechnol. Biochem. 2007, 71,309-322. [CrossRef] [PubMed]

34. Ali, Z.M.; Chin, L.H.; Marimuthu, M.; Lazan, H. Low temperature storage and modified atmosphere packaging of carambola fruit and their effects on ripening related texture changes, wall modification and CI symptoms. Postharvest Biol. Technol. 2004, 22, 181-192. [CrossRef]

35. Pagamas, P.; Nawata, E. Sensitive stages of fruit and seed development of chili pepper (Capsicum annuum L. var. Shishito) exposed to high-temperature stress. Sci. Hortic. 2008, 117, 21-25. [CrossRef]

36. Tully, R.E.; Musgrave, M.E.; Leopold, A.C. The seed coat as a control of imbibitional chilling injury. Crop Sci. 1981, 21, 312-317. [CrossRef]

Publisher's Note: MDPI stays neutral with regard to jurisdictional claims in published maps and institutional affiliations.

(C) 2020 by the authors. Licensee MDPI, Basel, Switzerland. This article is an open access article distributed under the terms and conditions of the Creative Commons Attribution (CC BY) license (http://creativecommons.org/licenses/by/4.0/). 